\title{
Combined Prediction of Wind Power with Chaotic Time Series Analysis
}

\author{
Wang Qiang* and Yang Yang
}

College of Electrical Engineering \& New Energy, China Three Gorges University, Yichang Hubei, 443012, China

\begin{abstract}
Wind power prediction is one of the most significant technologies to promote the capability of the whole power system that takes in wind electricity. A combined model for wind power forecasting is presented to decrease the influence of reconstructed parameters by chaotic time series analysis and the neural networks (NNs) in this work. The combined model respectively makes use of linear weighted model and NNs method to achieve combination of several neural networks models through phase space reconstruction after wind power series chaotic characteristics acquisition, which can integrate information and reduce prediction error in different embedding dimension, leading to higher forecast accuracy. Simulation is performed to the real power time series from Meijia wind farm. The results show that the proposed model is more effective than single embedding dimension model and linear weighted combination model, and the prediction error of neural network combination is less than $7 \%$.
\end{abstract}

Keywords: Chaotic time series analysis, embedding dimension, neural network combination, phase space reconstruction, wind power prediction.

\section{INTRODUCTION}

Wind resource has been paid more and more attention around the world because of global energy shortage and serious pollution. The power of wind possesses excellent characteristics such as sustainability, limitlessness, and cleanness. Wind energy has been competing with conventional fossil fuel source by operating successfully large size wind turbines. Nevertheless, Wind energy production is uncertain due to its intermittence and randomness. The overmuch increment of wind power connected capability can make power supply system unstable, and will lead to larger spinning reserve capacity that enhances the whole operation cost. Therefore, short-term and middle-term wind power prediction for electricity power schedule and operation of wind turbines [1,2], should be done to promote the competiveness with other energy forms in a wholesale electricity market [3].

The current methods of short-term wind power prediction are classified three catalogues in $[4,5]$ which are physical method, statistical method and intelligent learning. Physical method needs a large number of history data, but effective digital weather prediction data and the physical information around the farm must be available. Statistical method [6] is simple relatively, using uniform data, nevertheless, it can't deal with emergency information well. Intelligent learning may modify prediction model online in consistent with the wind farm position. Although intelligent learning model has been improved greatly in comparison with former ones, it is still hard to decide the network structure. Wind power

\footnotetext{
*Address correspondence to this author at the College of Electrical Engineering \& New Energy, China Three Gorges University, Yichang Hubei, 443012, China; Tel: 13872619030; Fax; 0086-717-6352670;

E-mail: sunjian2009@ctgu.edu.cn
}

forecasting based on time series was presented with error $32 \%$ [7]. The error of RBF neural network prediction [8] was $15.9 \%$. Numeric weather prediction (NWP) information was explored to forecast wind power with error $6.5 \%$ only, but there is not special NWP prediction model at home as yet, so weather data from abroad bring about extra cost.

Chaos theory opens up a new way for wind power prediction. History power can be reconstructed in phase space to resume the original dynamic system, based on embedding theorem proposed by Takens [9], which reduce blindness of network input numbers selected. Determination of embedding dimension and delay time is the foundation of phase space reconstruction. System with different embedding dimension can be reconstructed in different phase space, so different prediction results will be obtained. Combined prediction is presented in this work, utilizing comprehensively the information from system with different embedding dimensions, and reducing the probability of large prediction error with single model. Experimental results demonstrate that the approach proposed in this paper has better accuracy for short-term wind power prediction than linear weighted combination and single embedding model. Simultaneously, it can instruct the selection of neural network structure.

The work consists of five sections. The estimation calculation of delay time and embedding dimension is performed to extract the wind power chaotic features in section 2. Section 3 discusses the establishment of prediction models, including linear combination prediction and neural network combination prediction based on phase space reconstruction. Section 4 uses an actual case to compare the proposed approach with single neural network prediction . Concludes are made for this paper in section 5 . 


\section{PHASE SPACE RECONSTRUCTION ON CHAOS THEORY}

As for a chaotic system, the phase space can be used to reconstruct a univariate time series, because all the variable information in this dynamic system may be contained in the univariate time series [9]. The fundamental of phase space reconstruction is Taken's delay embedding theorem, which is described that if appropriate delay time $\tau$ and embedding dimension $m$ are selected, where $m>2 * d+1$ is satisfied generally, the geometry characteristics of original chaotic dynamic system is equivalent with that of $m$ dimension reconstructed state space, and they have the same topological structure. Taken's delay embedding theorem lays the theoretic foundation for chaotic time series prediction. The Lyapunov exponent is another important parameter for time series which recognizes its chaotic characteristics.

For chaotic time series $x_{1}, x_{2}, \cdots, x_{n-1}, x_{n}$, phase space can be expressed as follows:

$$
\begin{aligned}
& Y_{m}(k)=[x(k), x(k+\tau), \cdots, x(k+(m-1) \tau)] \quad k=1,2, \cdots, N, \\
& N=n-(m-1) \tau
\end{aligned}
$$

Where $m$ and $\tau$ are embedding dimension and delay constant respectively.

For phase space reconstruction, embedding dimension and delay constant selection is very significant and difficult. The appropriate parameters $\tau$ and $m$ will affect the quality of phase space reconstruction directly, and come to influence the prediction accuracy.

\subsection{Calculation of Delay Time $\tau$}

Mutual information method [10] is an effective approach to estimate delay constant in the reconstructed phase space, used widely.

To introduce mutual information method briefly, chaotic time series $x_{1}, x_{2}, \cdots, x_{n-1}, x_{n}$ will be rewritten as the following evolution vectors:

$(x, y)=(x(k), x(k+\tau)), t=1, \ldots, n$

Mutual information $I_{x y}(\tau)$ is defined in formula (3).

$I_{x y}(\tau)=H(x)+H(y)-H(x, y)$

$$
H(x)=-\sum P_{x}(x) \log \left[P_{x}(x)\right],
$$

Where $H(y)=-\sum P_{y}(y) \log \left[P_{y}(y)\right]$,

$$
H(x, y)=-\sum P_{x y}(x, y) \log \left[P_{x y}(x, y)\right]
$$

Where $H(\cdot)$ is the entropy function which is dependent on $P_{x}(x), P_{y}(y)$ and $P_{x y}(x, y)$, probability density distribution of $x, y$ and joint probability density distribution of $x$ and $y$ respectively. The value of $I_{x y}(\tau)$ reflects the correlation strength between $x$ and $y$. The smaller $I_{x y}(\tau)$ is, the weaker the correlation between $x$ and $y$ is. Too weak corre- lation between $x$ and $y$ may lead to uncertain system dynamics, so delay time $\tau$ can't be too large. The best choice of $\tau$ should be the one that brings the first minimum $I_{x y}(\tau)$.

\subsection{Estimation the Embedding Dimension}

Correlation dimension method $[11,12]$ is adopted to reckon the embedding dimension of a given time series. The correlation dimension rely on the correlation integral defined as follows:

$C(r)=\frac{1}{M^{2}} \sum_{i, j=1}^{M} \theta\left(r-r_{i j}\right), r_{i j}=\|x(i)-y(j)\|$

Where $\theta(\cdot)$ is the Heaviside step function:

$\theta(x)=\left\{\begin{array}{l}0, x \leq 0 \\ 1, x>0\end{array}\right.$

Evidently, the correlation integral $C(r)$ is determined by the size of $r$. When $r$ is larger than $r_{i j}, C(r)=1$; When $r$ is smaller than $r_{i j}, C(r)=0$. However, none of the above cases can exhibit all inner properties of the dynamic system, so it is important to choose an appropriate $r$ according to different parameters. When $r \rightarrow 0$, the relation between the correlation integral $C(r)$ and $r$ should be $\lim _{r \rightarrow 0} C(r) \propto r^{d}$, then an appropriate $r$ is decided. The correlation dimension $d$ is given by

$d=\frac{\log (C(r))}{\log (r)}$

Therefore, the embedding dimension $m$ can be computed from $m=\operatorname{int}(2 * d+1)$ as discussed above.

\subsection{Computation the Largest Lyapunov Exponent}

The Lyapunov spectrum analysis is illustrated to calculate the largest Lyapunov exponent in this section [13], which is presented on the fact that the motion orbit of chaotic system is hypersensitive to its initial status. The difference between two adjacent orbits exponentially enlarges with time transition, which can be quantized by the Lyapunov exponent. In the process of chaotic characteristics analysis for wind power time series, the whole Lyapunov exponent spectrum is not required, and if only the largest Lyapunov exponent is positive, the time series is identified as a chaotic system. To calculate the largest Lyapunov exponent, an algorithm is presented by Wolf and others [14], based on the movement of phase trajectory, phase plane and phase volume. As mentioned above, a reconstructed time series with embedding dimension $m$ and delay constant $\tau$ can be expressed as follows:

$p(t)=[x(t), x(t+\tau), \ldots, x(t+(m-1) \tau)]^{T}$

Where $t=1,2, \ldots, M$. 


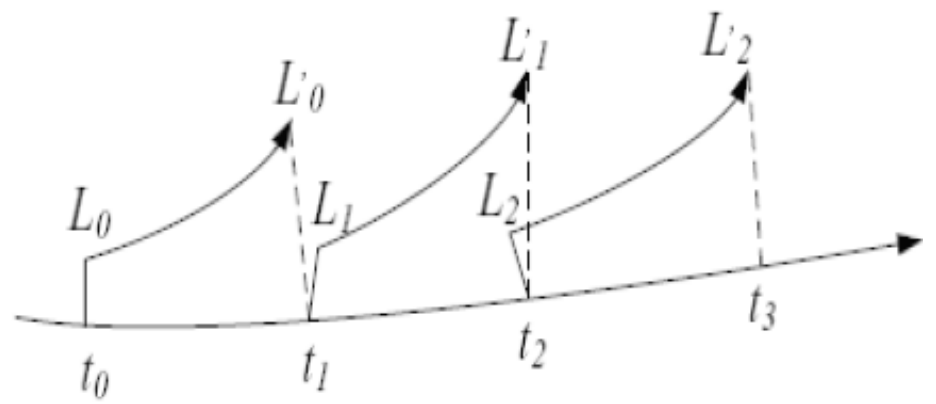

Fig. (1). Calculation of Lyapunov exponent of a time series.

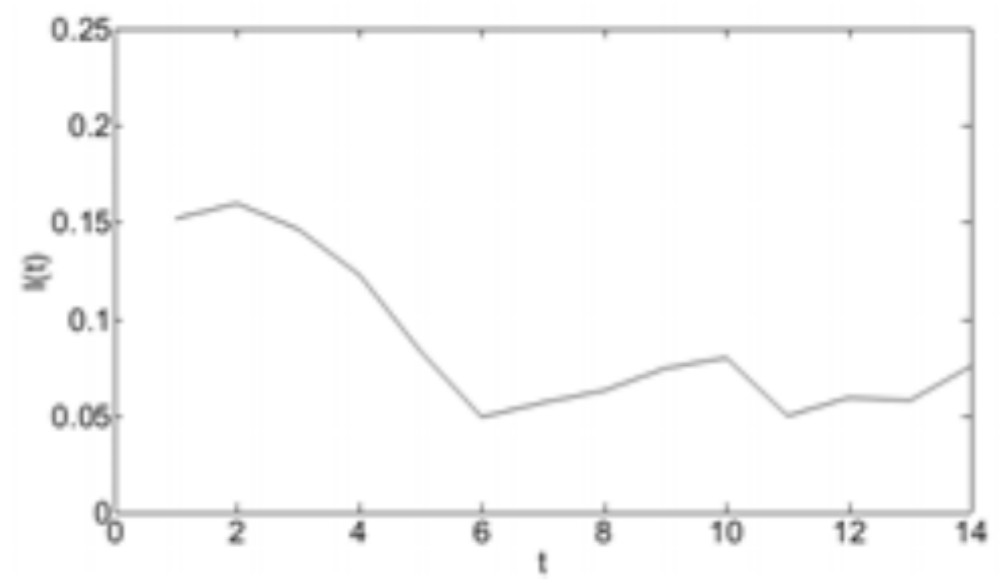

Fig. (2). The curve of mutual information function.

Set initial time $t_{0}$, terminal time $t_{M}$. And $M=N-(m-1) \tau, N$ is the terminal point of the time series. Give the initial point of reconstructed time series $p\left(t_{0}\right)$, and suppose the nearest neighboring point of $p\left(t_{0}\right)$ is $p_{0}\left(t_{0}\right)$, the distance between $p\left(t_{0}\right)$ and $p_{0}\left(t_{0}\right)$ is denoted as $L_{0}$. Track the time evolution of these two points forward to $t_{i}$. Once the distance becomes bigger than the predetermined threshold $\varepsilon(\varepsilon>0)$, that is $L_{0}^{\prime}=\left|p\left(t_{1}\right)-p\left(t_{0}\right)\right|>\varepsilon$, then retain $p\left(t_{1}\right)$, and search the nearest neighbor $p_{1}\left(t_{1}\right)$ of $p\left(t_{1}\right)$ which satisfies $L_{1}=\left|p\left(t_{1}\right)-p_{1}\left(t_{1}\right)\right|<\varepsilon$. The steps mentioned above should be iterated until $p(t)$ reaches terminal point $N$, shown as Fig. (1). The number of iterations would be $t_{M}-t_{0}$. And finally, we can get the largest Lyapunov exponent as follows:

$\lambda=\frac{1}{t_{M}-t_{0}} \sum_{i=1}^{M} \ln \frac{L_{i}^{\prime}}{L_{i}}$

Where, $L_{i}^{\prime}=\left|p\left(t_{i}\right)-p\left(t_{i-1}\right)\right|, L_{i}=\left|p\left(t_{i}\right)-p_{i}\left(t_{i}\right)\right|$, and $p_{i}\left(t_{i}\right)$ is a point in neighborhood with radius $r=\varepsilon$ of $p\left(t_{i}\right)$ at the time $t_{i}$.

\subsection{Characteristic Analysis of Wind Power Time Series in the Real World}

In Meijia wind farm, generation power is sampled at 30 min intervals at a $3.0 \mathrm{MW}$ wind turbine(randomly chosen) in a duration of two months. For the chosen wind turbine, the cut-in speed is $3 \mathrm{~m} / \mathrm{s}$, the rated speed is $15 \mathrm{~m} / \mathrm{s}$, and the cut-out speed is $22 \mathrm{~m} / \mathrm{s}$. In the sight of turbine operations, wind speed in the range $[3 \mathrm{~m} / \mathrm{s}, 15 \mathrm{~m} / \mathrm{s}$ ] is of interest to industry. As a result, the data with a wind speed out of range [3 m/s, $15 \mathrm{~m} / \mathrm{s}$ ] have been eliminated from analysis in this paper. Sample points with a negative power output is not in the scope of this article. $2 / 3$ of all data is used to build models, and the leavings is explored to test and validate models.

The delay time and embedding dimension are calculated respectively through the approaches mentioned above, which are shown in Fig. (2) and Fig. (3).

From Fig. (2), we can see that mutual information function get its minimum first, when $\tau=6$, so the value of delay time is 12. Fig. (3) shows the slope of $\ln (C(r))$ versus $\ln (r)$ when $m$ is varying from 2 to 15 . As displayed in Fig. (3), the curves are close to straight lines when $r$ changes in the range $[0.1,0.2]$ and $m$ in $[2,15]$, and their slop is approximately equal to each other. Thus the correlation dimension $d$ can be evaluated by averaging the slopes of these 14 curves, consequently $d=3.51$. From $m=\operatorname{int}(2 * d+1)$, we can obtain $m=8$. After $\tau$ and $m$ being evaluated, the largest Lyapunov exponent can be estimated, and the result 


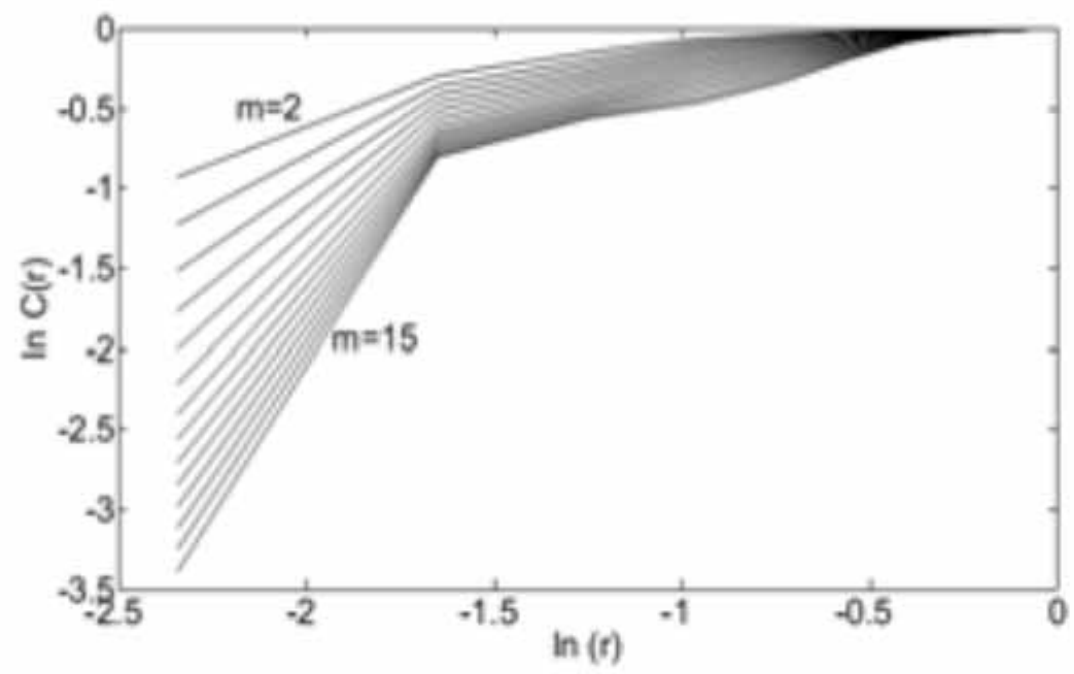

Fig. (3). The slop of $\ln (C(r))$ versus $\ln (r)$ with different $m$ gives the correlation dimension $d$ of the wind speed time series.

is a positive number 0.2548 , which verfy that wind power time series is a chaotic system.

\section{ESTABLISHMENT OF PREDICTION MODEL}

\subsection{Single Neural Network Prediction Model Based Phase Space Reconstruction}

Suppose that $x(k)(k=1,2, \cdots, n)$ is a sampled time series, which has the status transfer formals in phase space as follows:

$Y_{m}(k+1)=f\left(Y_{m}(k)\right)$

According formula (1), formula (11) is followed through extending formula (10) as:

$[x(k+1), \cdots, x(k+1+(m-1) \tau]=f([x(k), \cdots, x(k+(m-1) \tau])$

Where the last component $x(t+1+(m-1) \tau)$ is unknown, then formula (12) can be rewritten as:

$x(k+1+(m-1) \tau)=F(x(k), \cdots, x(k+(m-1) \tau))$

Where $F(\cdot)$ is a real function from $m$ dimension to 1 dimension. For wind power generation system, $F(\cdot)$ is a nonlinear function, expressed hardly, so nonlinear mapping capability is explored to solve it. Simultaneously, $m$ components of phase points in embedding space should be chosen neural network inputs, which can avoid selection randomness of input units and information missing [15].

\subsection{Combined Prediction of Wind Power}

Combination prediction method [16] is built on maximization of information utilization, and optimize to combine information from multiple models. It synthesizes the merits of different models, and promotes prediction accuracy obviously. Because embedding dimension can be calculated by various approaches, and affected by human factors, different embedding dimensions would be obtained. Therefore, time series may be reconstructed to different phase space, which leads to difference among prediction results. In order to improve stability and accuracy of prediction model, combined prediction based multi-dimension embedding phase space is utilized to forecast wind power.

1) Linear combination prediction

Assume $x(t)(t=1,2, \cdots, n)$ is real value at $t$ moment, prediction values under different embedding dimensions are $\hat{x}_{1}(t), \hat{x}_{2}(t), \cdots, \hat{x}_{p}(t)$ respectively, linear combination prediction result will follow as:

$\hat{x}(t)=\mathrm{a}_{1} \hat{x}_{1}(t)+\mathrm{a}_{2} \hat{x}_{2}(t)+\cdots+\mathrm{a}_{p} \hat{x}_{p}(t)$

Where $\mathrm{A}=\left(\mathrm{a}_{1}, \mathrm{a}_{2}, \cdots, \mathrm{a}_{p}\right)^{T}$ is weight coefficient vector, and satisfied with:

$a_{1}+\mathrm{a}_{2}+\cdots+\mathrm{a}_{p}=1$

When embedding dimension is $i$, model prediction error at $t$ moment is defined as:

$e_{i t}=x(t)-\hat{x}_{i}(t), \quad i=1,2, \cdots, p \quad t=1,2, \cdots, n$

Relevant prediction error information matrix is:

$E=\left[\left(e_{i t}\right)_{p \times n}\right]\left[\left(e_{i t}(t)\right]^{T}\right.$

Then error square sum of linear combination prediction can be deduced as formula (18):

$J=\sum_{t=1}^{n}[x(t)-\hat{x}(t)]^{2}=\sum_{t=1}^{n}\left(\sum_{i=1}^{p} a_{i} e_{i t}\right)^{2}=A^{T} E A$

The minimization of error square sum is chosen as objective function. weight coefficient vector A can be decided through solving the following optimization problem.

$$
\begin{array}{ll}
\min & J=\mathrm{A}^{T} E \mathrm{~A} \\
\text { s.t. } & R^{T} \mathrm{~A}=1, R=(1,1, \cdots 1)^{T}
\end{array}
$$




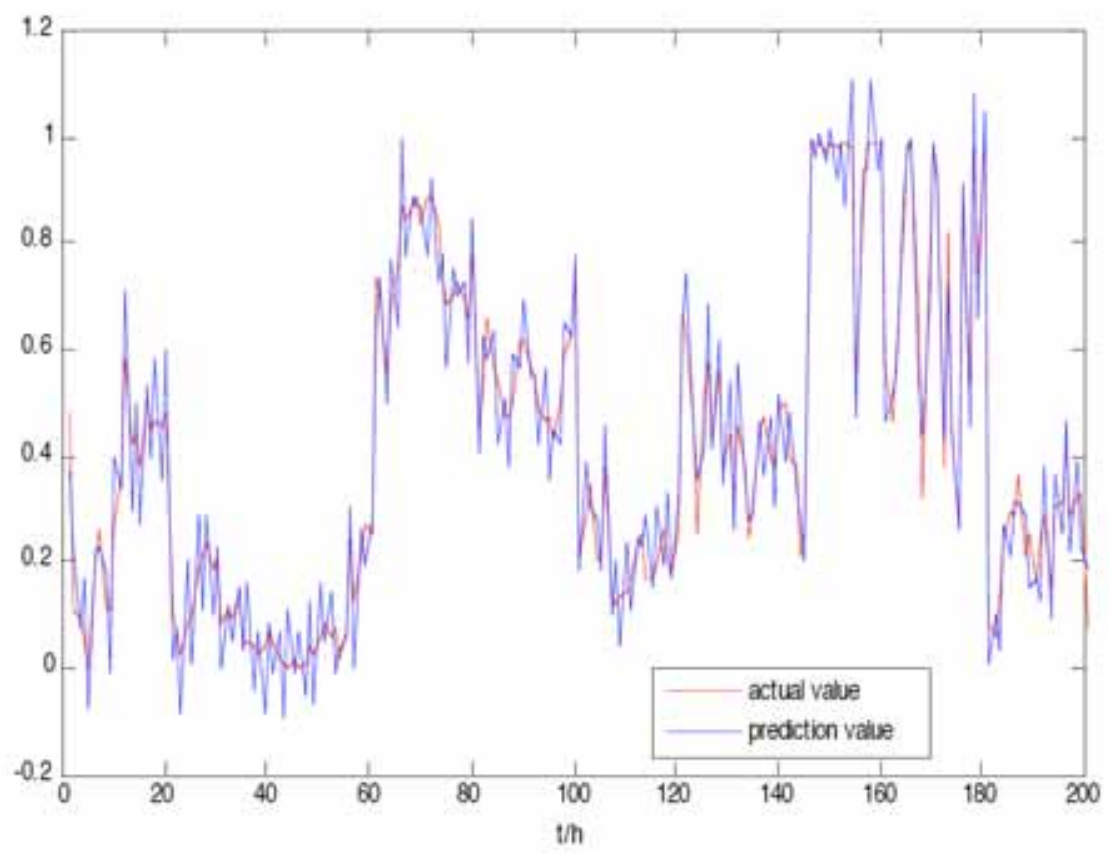

Fig. (4). Prediction values and actual values with $m=8$.

Namely, the optimal solutions of second order programming model are the best weights of linear combination prediction model.

2) Combination prediction based neural network

Linear combination prediction mentioned above is called fixed weight combination prediction method, where weights are unchanged with the varied environment, so prediction accuracy will be affected. If formula (19) is satisfied by $\hat{x}(t)$, and $\phi(\cdot)$ is a nonlinear function, then this combination prediction is named nonlinear combination prediction, where weights are variable.

$\hat{x}(t)=\phi\left(\hat{x}_{1}(t), \hat{x}_{2}(t), \cdots, \hat{x}_{p}(t)\right)$

Explicitly, nonlinear combination prediction can reflect the nonlinearity of system, then it is more reasonable than fixed weight combination prediction.

In recent years, it is a great breakthrough to apply neural network to combination prediction [17]. In this paper, three layer BPNN is designed, whose inputs are prediction values from models with different embedding dimensions, trained continually through gradient descent method to modify weights, finally, nonlinear function $\phi(\cdot)$ is established between inputs and outputs.

\section{ACTUAL WIND POWER PREDICTION}

From Meijia wind farm case in 2.4 section, delay time $\tau=6$, and embedding dimension $m=8$, therefore, three neural network prediction models based phase space reconstruction are established with $m=7,8$ and 9 in this paper, respectively, which are surrounding embedding dimension.
For neural network models, the inputs are actual data at some phase point, that is $\{x(i), x(i+\tau), x(i+2 \tau)$, $\cdots, x(i+(m-1) \tau)\}$, and outputs are prediction values at next interval. For the computation resolution and convenience, the data is normalized to the range $[0,1]$ firstly. Length limited, only 200 prediction results with $m=8$ are show in Fig. (4).

In order to improve wind power prediction accuracy and model stability, linear combination and neural network are utilized to assemble results from three models built above. Quadratic programming function quadprog in Matlab optimization toolbox is used to solve the model as formula (18), and linear weight coefficients are achieved as 0.0128, 0.7806 and 0.2065 , respectively. The prediction results with linear combination are shown in Fig. (5). The prediction results with three different dimensions are considered as inputs of forward neural network, and prediction power as outputs, and weights of various prediction approaches for combination model are acquired with self-learning. The architecture of neural network combination is determined as 3-8-1 by trial and error. The prediction results with neural network combination are shown in Fig. (6). Because there is a direct relationship between prediction error and capability of wind turbine generator, absolute average error should be normalized to evaluate prediction performance. Table $\mathbf{1}$ gives out prediction error of various models.

From Fig. (4-6) and Table 1, we can conclude 1) all models track actual wind power change, especially neural network prediction results coincide with actual values best; 2) prediction error is the smallest when embedding dimension is 8 , so its prediction result has the largest weight in linear combination model; 3 ) the prediction performance of 

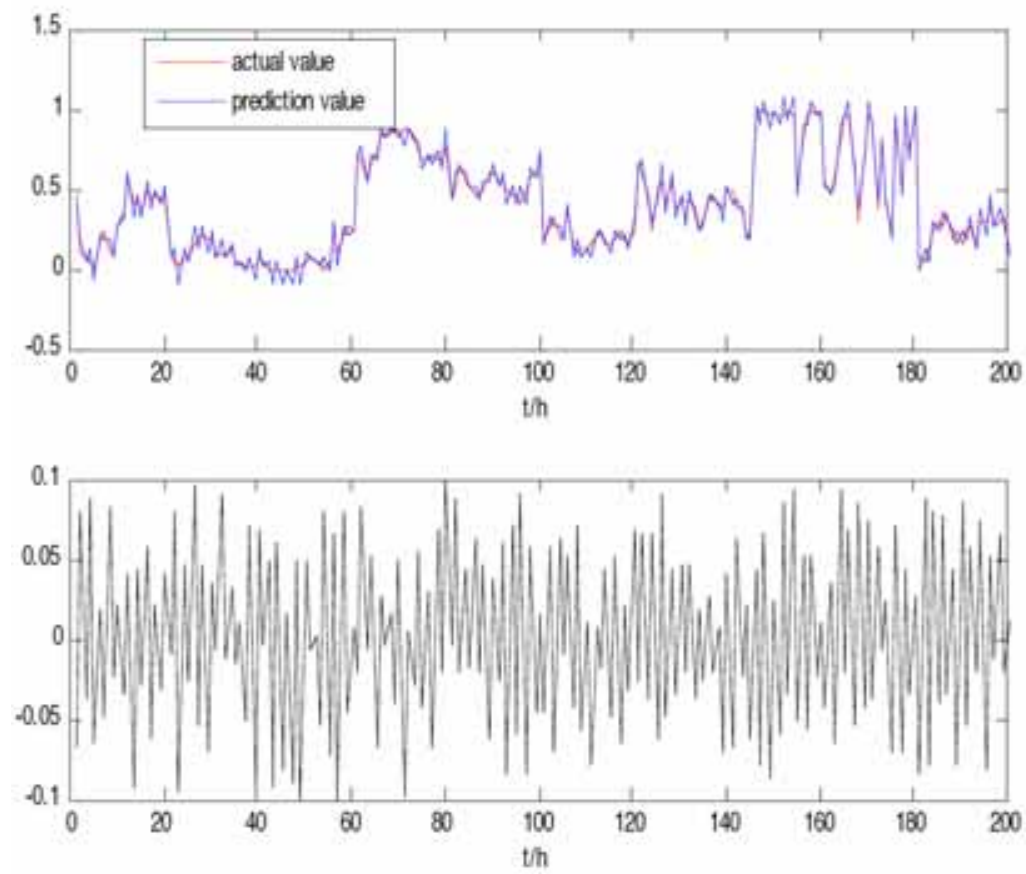

Fig. (5). The curves of linear combination prediction and error.
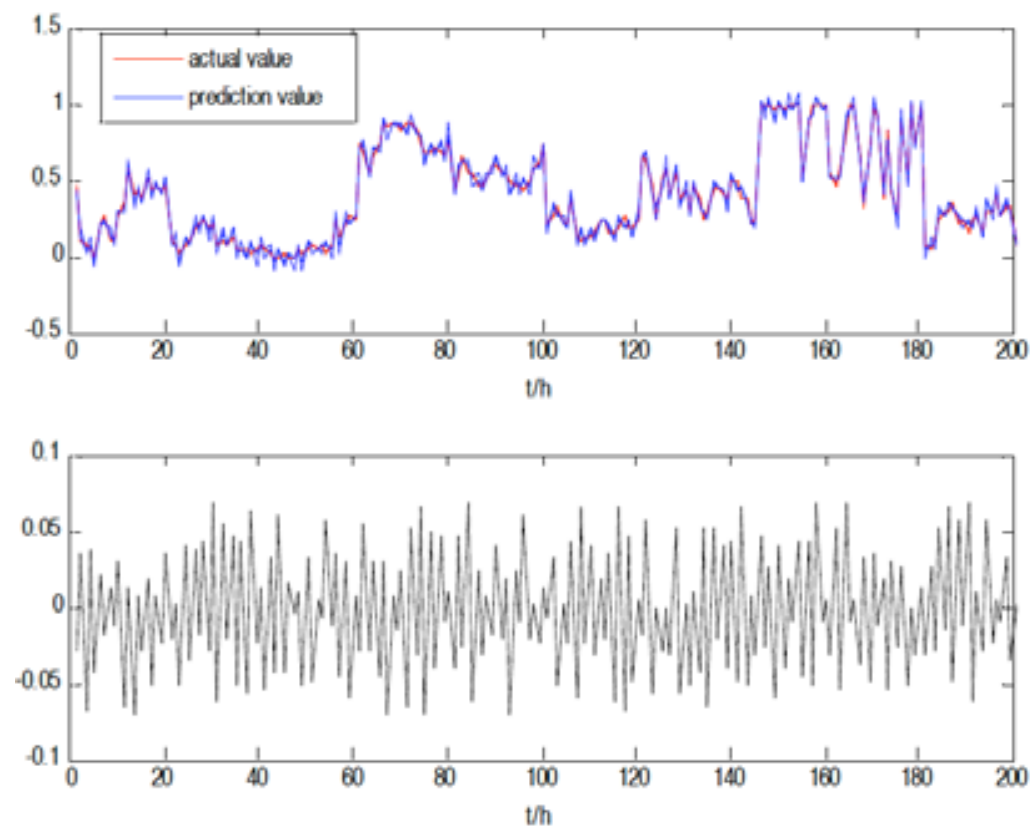

Fig. (6). The curves of Neural network combination prediction and error.

combination prediction with multi-dimension embedding phase space is better than neural network model with single phase space reconstruction; 4) embedding dimension, namely, the input number for neural network has great impact on the prediction accuracy; 5) compared with linear combination, neural network combination prediction has higher accuracy; 6) among all models, the error of neural network combination prediction is the smallest, and its absolute average error is $6.70 \%$.

\section{CONCLUSION}

Chaotic system may contain different information after the reconstruction using different embedding dimensions. In order to improve the system stability and prediction accuracy, and decrease the prediction deviation with different embedding dimensions, combined prediction model embedding multi-dimension is built in this paper based on chaotic time series analysis. Weighted linear combined prediction is 
Table 1. Comparison of prediction error with various models.

\begin{tabular}{|c|c|}
\hline Prediction Model & Absolute Average Error \% \\
\hline \hline$m=7$ & 7.11 \\
\hline$m=8$ & 6.90 \\
\hline$m=9$ & 6.92 \\
\hline Linear combination & 6.86 \\
\hline Neural network combination & 6.70 \\
\hline
\end{tabular}

simple relatively, however, it can't show the nonlinearity of wind power system precisely, which affects the prediction accuracy. Whereas, neural network nonlinear combination forecasting is able to reveal the nonlinearity of wind power system better, whose combination functions can be solved easy, so it is fitful to forecast for chaotic systems.

In the aspect of time horizon, the proposed combination model benefits very short-term wind power prediction for power system operation and control, because it doesn't relate with numeric weather prediction information, and can trace with wind power rapidly.

\section{CONFLICT OF INTEREST}

The authors confirm that this article content has no conflict of interest.

\section{ACKNOWLEDGEMENTS}

This work is supported by the National Natural Science Foundation of China (Grant No. 51277110), and the Natural Science Foundation of Hubei Province Department of Education (Grant No. Q20121305), and Science Technology Research and Development Fund of Yichang Municipality (Grant No. A2012-302-14 , A2012-302-15).

\section{REFERENCES}

[1] A. Kusiak, H. Zheng and Z. Song, "Wind farm power prediction: a data-mining approach", Wind Energy, vol. 12, no. 3, pp. 275-293, 2009.
[2] L. Ma, S. Luan, C. Liang, H. Liu and Y. Zhang, "A review on the forecasting of wind speed and generated power", Renewable and Sustainable Energy Reviews, vol. 13, no. 4, pp. 915-920, 2009.

[3] S. Ismael, "Short-term prediction of wind energy production", International Journal of Forecasting, vol.22, no. 1, pp.43-56, 2006.

[4] G. Kariniotakis, P. Pinson, N. Siebert, G. Giebel and R. Barthelmie, "The state of the art in short-term prediction of wind powerfrom an offshore perspective", Anemos Project Report D1.1 (Available online), http://anemos.cma.fr; 2003.

[5] B. Ernst, B. Oakleaf and M.L. Ahlstrom, "Predicting the wind", IEEE Trans. On Power and Energy Magazine, vol. 5, no. 6, pp. 7989, 2007.

[6] S. Christos, K. Akrivi, G. George and K. George, "Wind power prediction based on numerical and statistical models", Wind Engineering and Industrial Aerodynamics, no. 112, pp. 25-38, 2013.

[7] X.Y. Yang, Y. Xiao and S.Y. Chen, "Wind speed and generated power forecasting in wind farm", Proc of the CSEE, vol. 25, no. 11, pp.1-5, 2005.

[8] S. Han, Ed, "Study of short-term wind power prediction method", Ph.D. thesis, North China Electric Power University, Beijing, China, 2008.

[9] F. Takens, "Detecting strange attractors in turbulence", Lecture Notes in Mathematics, vol. 898, pp. 366-381, 1981.

[10] L. Dong, S. Gao, X.Z. Liao, "Chaos characteristic analysis on the time series of wind power generation capacity", Acta Energiae Solaris Sinica, vol. 28, no. 11, pp.1290-1294, 2007.

[11] Y.J. Lu, X.W. Tang, Y. Zhang, "Chaotic property of pricing of supply chain production and its price forecasting", Control and Decision, vol. 21, No. 4, 2006, pp. 445-448.

[12] M.B. Kennel, R. Brown, and H.D.L. Abarbanel, "Determining embedding dimension for phase space reconstruction using a geometrical construction", Physical Review A, Vol. 45, No. 6, 1992 pp.3403-3411.

[13] R. Shaw, "Strange attractors, chaotic behavior, and information flow", Z Naturf, vol. 36a, pp. 80-112, 1981.

[14] A. Wolf, J.B. Swift, H.L. Swinney and J.A. Vastano, "Determining Lyapunov exponent from a time series", Physica D, vol. 16, no. 2, pp. 285-317, 1985

[15] S.L. Lei, C.X. Sun and Q. Zhou, "Short-term load forecasting using one-rank local-region method in multi-dimension embedding phase space”, Power System Technology, vol. 29, no. 13, pp.45-49, 2005.

[16] H.Y. Chen, "Effect theory of combined forecast method and its application", Beijing: Science Press, 2008.

[17] G. Sideratos and N. Hatziargyriou, "Using radial basis neural networks to estimate wind power production". IEEE Power Energy Society General Meeting, vol.1, pp.1-7, 2007.

(C) Qiang and Yang; Licensee Bentham Open.

This is an open access article licensed under the terms of the Creative Commons Attribution Non-Commercial License (http://creativecommons.org/licenses/by-nc/3.0/) which permits unrestricted, non-commercial use, distribution and reproduction in any medium, provided the work is properly cited. 